OPEN ACCESS

Edited by:

Andrea Fiorillo,

Università degli Studi della Campania

"Luigi Vanvitelli" Naples, Italy

Reviewed by:

Francesco Bartoli,

Università degli studi di Milano

Bicocca, Italy

Steve Simpson $\mathrm{Jr}$

The University of Melbourne, Australia

Beth McGinty,

Johns Hopkins University,

United States

*Correspondence:

Antonio Ventriglio

a.ventriglio@libero.it

Specialty section:

This article was submitted to

Psychosomatic Medicine,

a section of the journal

Frontiers in Psychiatry

Received: 22 August 2018 Accepted: 17 December 2018 Published: 16 January 2019

Citation:

Ventriglio A, Baldessarini RJ, Vitrani $G$

Bonfitto I, Cecere AC, Rinaldi A,

Petito A and Bellomo A (2019)

Metabolic Syndrome in Psychotic

Disorder Patients Treated With Oral

and Long-Acting Injected

Antipsychotics.

Front. Psychiatry 9:744.

doi: 10.3389/fpsyt.2018.00744

\section{Metabolic Syndrome in Psychotic Disorder Patients Treated With Oral and Long-Acting Injected Antipsychotics}

\author{
Antonio Ventriglio ${ }^{1 *}$, Ross J. Baldessarini ${ }^{2,3}$, Giovanna Vitrani ${ }^{1}$, Iris Bonfitto ${ }^{1}$, \\ Angela Chiara Cecere ${ }^{1}$, Angelo Rinaldi ${ }^{1}$, Annamaria Petito ${ }^{1}$ and Antonello Bellomo ${ }^{1}$ \\ ${ }^{1}$ Department of Clinical and Experimental Medicine, University of Foggia, Foggia, Italy, ${ }^{2}$ International Consortium for \\ Psychotic \& Mood Disorder Research, McLean Hospital, Belmont, MA, United States, ${ }^{3}$ Department of Psychiatry, Harvard \\ Medical School, McLean Hospital, Boston, MA, United States
}

Background: Severe mental illnesses are associated with increased risks for metabolic syndrome (MetS) and other medical disorders, often with unfavorable outcomes. MetS may be more likely with schizoaffective disorder (SzAff) than schizophrenia (Sz). MetS is associated with long-term antipsychotic drug treatment, but relative risk with orally administered vs. long-acting injected ( $L A l)$ antipsychotics is uncertain.

Methods: Subjects ( $n=151$ with a DSM-IV-TR chronic psychotic disorder: 89 Sz, 62 SzAff), treated with oral or LAl antipsychotics were compared for risk of MetS, initially with bivariate comparisons and then by multivariate regression modeling.

Results: Aside from measures on which diagnosis of MetS is based, factors preliminarily associated with MetS included antipsychotic drug dose, "high-risk" antipsychotics associated with weight-gain, older age and female sex. Defining factors associated with diagnosis of MetS ranked in multivariate regression as: higher fasting glucose, lower LDL cholesterol, higher diastolic blood pressure, and higher BMI. Risk of MetS with antipsychotics ranked: quetiapine $\geq$ clozapine $\geq$ paliperidone $\geq$ olanzapine $\geq$ risperidone $\geq$ haloperidol $\geq$ aripiprazole. Other associated risk factors in multivariate modeling ranked: higher antipsychotic dose, older age, and SzAff diagnosis, but not oral vs. LAl antipsychotics

Conclusions: SzAff diagnosis and higher antipsychotic doses were associated with MetS, whereas orally vs. injected antipsychotics did not differ in risk of MetS.

Keywords: metabolic syndrome, antipsychotics, long-acting injected, schizoaffective, schizophrenia

\section{INTRODUCTION}

Persons with severe mental illnesses have increased risk for metabolic disorders, including metabolic syndrome (MetS), characterized by obesity, type 2 diabetes mellitus, dyslipidemia, and hypertension (1). Such disorders appear to be related to an unhealthy diet, lack of regular exercise, adverse effects of psychotropic drugs, and possibly to undefined risk factors associated with the illnesses themselves $(2,3)$. Much of the research on this topic has involved patients diagnosed with chronic psychotic or mood disorders, particularly schizophrenia (Sz) and bipolar disorder 
(2, 4-6). Few studies have compared physical health of subjects diagnosed with schizoaffective disorder (SzAff) to that of other patients with other psychotic-disorder diagnoses, including $\mathrm{Sz}$, but SzAff patients may have a greater risk of MetS than those with other major psychiatric disorders (6). SzAff patients are characterized by emotional and behavioral instability over time as well as psychotic features, and often are treated with relatively complex pharmacological regimens (7). Both emotional instability and complex treatments may contribute to an increased risk of metabolic disorders (1).

Also uncertain is whether specific types of medicines differ appreciably in their associations with risks of metabolic disorders. In particular, the extent to which relative metabolic risks of modern or second-generation antipsychotics (SGAs) and their long-acting injected (LAI) preparations differ from older or orally administered antipsychotics remains uncertain (1, 8-10).

The preceding considerations led us to compare clinical measures, in particular indices of metabolic health, among SzAff vs. Sz patient-subjects to identify factors associated specifically with MetS, including comparison of orally administered vs. LAI antipsychotics. We hypothesized that SzAff subjects would have a higher risk of MetS than Sz subjects, and that the risk might be lower with LAI antipsychotic treatments.

\section{METHODS}

From June 2014 to February 2017, we enrolled study subjects as part of a program monitoring the health of psychotic disorder patients attending the Day Hospital Service for Severe Mental Disorders in the Psychiatric Department at the University of Foggia Medical Center. A total of 151 consecutive patients were enrolled as study-subjects, including 89 diagnosed with $\mathrm{Sz}$ and 62 as SzAff by two expert clinicians (AB, AV) based on DSMIV-TR (Diagnostic and Statistical Manual of mental disordersText Revision) criteria (11). Treatments were selected clinically and included oral antipsychotics $(n=64$, with or without mood-stabilizers or antidepressants) as well as LAI antipsychotics ( $n=87$, usually as monotherapy).

All subjects provided written informed consent to participate, after study procedures approved by the University of Foggia medical center ethics committee were explained to them. Patients were enrolled in a stable phase of their illness and treatments; candidates who required psychiatric hospitalization, had revised treatment protocols within the previous 6 months, were actively abusing alcohol or drugs (confirmed by urine assays), or were pregnant, were excluded from the study.

Current psychiatric morbidity was assessed and rated with the Positive and Negative Syndrome Scale (PANSS) (12), and Brief Psychiatric Rating Scale (BPRS) (13) by two experienced psychiatrist-investigators $(\mathrm{AB}, \mathrm{AV})$. Raters were held unaware of treatments given, and their ratings yielded high, independent, interrater agreement $\left(\chi^{2} \geq 0.90\right)$. Being considered "mildly ill" corresponded to a PANSS total score of $\leq 58$ or BPRS score of $\leq 31$, "moderately ill" corresponded to PANSS ratings of 59-75 or BPRS scores of 32-40, "moderately severely ill" corresponded to PANSS of 76-95 or BPRS of 41-53, and "severely ill" corresponded to a PANSS of 96-116 or BPRS of 54-126 $(12,13)$.

We also collected data on: demographics (sex, age, employment status), current pharmacological treatments (oral or LAI antipsychotics, mood stabilizers [MSs], and antidepressants [ADs]), and their doses; anthropometric and metabolic measures (height $[\mathrm{cm}]$ and weight $[\mathrm{kg}]$ for body-mass index $[\mathrm{BMI}])$, systolic and diastolic blood pressure $(\mathrm{mm} \mathrm{Hg})$, pulse (beats/min); serum concentrations of fasting glucose (FBS; $\mathrm{mg} / \mathrm{dL}), \%$-glycated hemoglobin (Hgb-A1c), total cholesterol (mg/dL), low density lipoproteins (LDL; mg/dL), high density lipoproteins (HDL; $\mathrm{mg} / \mathrm{dL})$, triglycerides $(\mathrm{mg} / \mathrm{dL})$; waist circumference $(\mathrm{cm})$, electrocardiographic rate-corrected QT repolarization interval (QTc, msec); serum levels of prolactin (ng/dL), thyroid stimulating hormone (TSH, mIU/L), and free thyroxin and triiodothyronine. We also recorded adverse events associated with treatment, and rated treatment-adherence with the 30-item Drug Attitude Inventory (DAI-30) (14).

We rated subjects for the presence of MetS defined by current, revised International Diabetes Federation (IDF) criteria, American Heart Association and International Association for the Study of Obesity $(15,16)$. MetS required meeting $\geq 3$ of the following 5 criteria: [a] large waist circumference $(\geq 102 \mathrm{~cm}$ in men, $\geq 88 \mathrm{~cm}$ in women); [b] elevated serum triglycerides $(\geq 150$ $\mathrm{ng} / \mathrm{dL})$; [c] low HDL-cholesterol $(<40 \mathrm{mg} / \mathrm{dL}$ in men and $<50 \mathrm{in}$ women); high blood pressure ( $\geq 130 \mathrm{~mm} \mathrm{Hg}$ systolic or $\geq 85 \mathrm{~mm}$ diastolic); elevated glucose as fasting blood sugar (FBS $>100$ $\mathrm{mg} / \mathrm{dL})$.

To facilitate comparisons, we converted antipsychotic doses to approximate oral daily mg-chlorpromazine-equivalents (CPZeq); LAI antipsychotic doses were estimated as total mg doses per days of injection cycles for conversion to CPZ-eq $(17,18)$. For MSs, we converted dosages to approximate daily mg-equivalents of lithium carbonate (Li-eq) $(18,19)$. Antidepressants were noted as being prescribed or not.

We compared measures collected among subjects diagnosed with SzAff and Sz, treated with LAI and oral antipsychotics, emphasizing comparisons of subjects with vs. without MetS. Data analyses used commercial statistical programs (Statview5, SAS Corp., Cary, North Carolina, USA for spreadsheets; Stata.13.0, Stata Corp., College Station, Texas, USA). Data are presented as means \pm standard deviation (SD) or with $95 \%$ confidence intervals (CI), or as percentages (\%), unless stated otherwise. Continuous data were compared using nonparametric Mann-Whitney rank-sum test ( $z$-score) to avoid problems of non-normal distribution of values, and categorical data were tested with contingency tables $\left(\chi^{2}\right)$. Factors yielding $p<0.10$ in preliminary bivariate comparisons were considered in multivariate logistic regression modeling, with presence of MetS as the outcome measure.

\section{RESULTS}

\section{Sample Characteristics and Treatments}

The 151 patient-subjects were aged $42.1 \pm 12.4$ years; $52.9 \%$ were men, $18.5 \%$ were employed. Diagnoses included Sz $(n=89$; $58.9 \%)$ and SzAff $(n=62 ; 41.1 \%)$. More men than women were 
diagnosed with $\mathrm{Sz}\left(\chi^{2}=6.76 ; p=0.009\right)$. Treatments included oral antipsychotics in $42.3 \%$, and LAI antipsychotics in $57.7 \%$ (none received both). Antipsychotics were combined with moodstabilizers (MSs) in only $14.5 \%$, or with antidepressants (ADs) in $12.3 \%$ (ranking by use: duloxetine $>$ paroxetine $>$ citalopram or $S$-citalopram $>$ sertraline). Both adjunctive treatments were given selectively with oral antipsychotics, by 6.4- (MSs) or 7.0times more (ADs; both $p \leq 0.006$ ) among SzAff than Sz subjects. Antipsychotic doses averaged $313 \pm 329 \mathrm{mg} /$ day CPZ-eq, and MS (carbamazepine, lithium carbonate, sodium valproate) total daily Li-eq doses averaged $650 \pm 244 \mathrm{mg}$. Overall, clinical ratings averaged $75.0 \pm 34.7$ for PANSS and $51.6 \pm 23.6$ for BPRS; both indicate moderate symptomatic severity, even though all patients reported clinical and treatment stability for at least six continuous preceding months. Prolonged, stable dosing assured that even the LAI antipsychotics were at pharmacokinetic steady-state.

Subjects who received LAI vs. oral antipsychotics had significantly lower levels of symptomatic morbidity. PANSS scores were, respectively, $58.0 \pm 27.6$ vs. $98.1 \pm 29.9$, and BPRS scores averaged $40.1 \pm 15.0$ vs. $67.1 \pm 24.5$ ( $z$-scores $=7.76$ and 6.85 , both $p<0.0001$ ).

No subject was considered to have a substance-use disorder, as was supported by urine drug assays, consistent with current substance abuse as an exclusion criterion. Adherence to prescribed treatments was considered good, as supported by a DAI-30 score of $9.64 \pm 2.19$ (of a total maximum of 30). There was a moderate rate of reported, treatment-associated, adverse events (19.8\%), most of which involved motor slowing or mild tremor.

\section{Risk and Measures Associated With Metabolic Syndrome}

Of the entire sample, $31.8 \%$ met diagnostic criteria for MetS (Table 1): $42.3 \%$ of women and $22.5 \%$ of men. Overall, BMI averaged $27.7 \pm 5.72 \mathrm{~kg} / \mathrm{m}^{2}$, in the nearly obese range. However, $35.8 \%$ of subjects $(38.0 \%$ of women and $33.8 \%$ of men) had BMI of $\geq 28.8 \mathrm{~kg} / \mathrm{m}^{2}$, taken to indicate obesity $(15,16)$.

Other factors possibly associated with MetS included: female sex, older age, SzAff vs. Sz diagnosis, higher BPRS psychosis score (which was associated with greater APD doses: Spearman $r_{s}=0.252$, slope $\left.=1.68[0.653-2.72], p=0.002\right)$, treatment with antipsychotics with relatively high risk of obesity and MetS (clozapine, olanzapine, paliperidone, quetiapine; Table 2), higher CPZ-eq antipsychotic dose, but not orally administered vs. LAI antipsychotics (Table 1). Additional metabolic and cardiovascular measures did not differ between subjects with vs. without MetS, including assays of TSH and thyroid hormones, prolactin, pulse rate, and ECG repolarization interval (QTc), nor did BPRS or PANSS ratings of psychosis-severity differ (Table 1).

As expected, measures that contributed to its diagnosis were highly deviant among subjects with MetS, including obesity, waist circumference, blood pressure, FBS, hemoglobin glycation, serum concentrations of cholesterol (higher total and LDL, lower HDL) and triglycerides (Table 1). BMI was not used to define MetS but was markedly elevated in subjects with MetS (Table 1). We also tested the strengths of association of such measures with the diagnosis of MetS using logistic regression modeling (Table 2). These ranked as: higher FBS $\geq$ lower HDL $\geq$ higher diastolic blood pressure $\geq$ higher BMI $\geq$ female sex (BMI and sex were not included in diagnostic criteria for MetS).

\section{Treatments and Metabolic Syndrome}

LAI antipsychotics were more prescribed than oral agents (57.7\% vs. $42.3 \%)$, particularly among Sz vs. SzAff subjects (65.5\% vs. $\left.34.5 \% ; \chi^{2}=3.67, p=0.055\right)$, whereas oral agents were used in half of both diagnostic groups. Average CPZ-eq daily doses of oral and LAI antipsychotics among $\mathrm{Sz}(295 \mathrm{mg})$ and SzAff subjects $(338 \mathrm{mg})$ did not differ significantly. LAI paliperidone palmitate was the most prescribed antipsychotic agent in both diagnostic groups ( $\mathrm{Sz} 34.8 \%$; SzAff $27.4 \%$; $31.8 \%$ overall); other LAI antipsychotic usage ranked: risperidone extended release $(10.6 \%)>$ aripiprazole long acting $(9.28 \%)>$ olanzapine palmitate $(4.64 \%)$. Usage of oral antipsychotics ranked: risperidone (Sz 11.2\%, SzAff 12.9\%, $11.9 \%$ overall $)>$ haloperidol $(7.28 \%$ overall $)=$ olanzapine $(7.28 \%)>$ aripiprazole $(5.30 \%)>$ quetiapine $(3.98 \%) \geq$ paliperidone $(3.31 \%)=$ clozapine $(3.31 \%)>$ ziprasidone $1.32 \%$.

Several metabolic measures were somewhat more favorable with use of LAI vs. oral antipsychotics, including total cholesterol (192 vs. $223 \mathrm{mg} / \mathrm{dL}$ ), LDL cholesterol (125 vs. $175 \mathrm{mg} / \mathrm{dL}$ ), triglycerides (148 vs. $188 \mathrm{mg} / \mathrm{dL})$; waist circumference $(103 \mathrm{~cm}$ [40.6 in] vs. $117 \mathrm{~cm}$ [46.1 in]); the cardiac QTc repolarization interval (399 vs. $413 \mathrm{msec}$ ); and circulating prolactin ( 42.7 vs. 61.3 $\mathrm{ng} / \mathrm{dL})$.

We compared the prevalence of MetS among subjects treated with different antipsychotic agents. Relatively high-risk drugs were quetiapine $(83.3 \%)$, clozapine $(60.0 \%)$, paliperidone $(34.0 \%)$ and olanzapine (33.4\%; Table 3). Of note, these risks were not accounted for by dose as prevalence of MetS and CPZ-eq doses were not significantly correlated (Table 3 ).

\section{Multivariable Modeling: Factors Associated With Metabolic Syndrome}

We used multivariable logistic regression modeling to identify factors associated independently with MetS. In order of significance, associated factors ranked: CPZ-eq antipsychotic dose, older age, and SzAff $>\mathrm{Sz}$ diagnosis, but not oral vs. LAI antipsychotics (Table 4).

\section{DISCUSSION}

This study involved 151 patient-subjects with chronic psychotic disorders who had been clinically stable on constant medication regimens for at least 6 months. LAI antipsychotics were given to $57.7 \%$, and oral agents to $42.3 \%$. LAI agents were more often given to $\mathrm{Sz}$ subjects, whereas use of oral antipsychotics was similarly prevalent in both SzAff and Sz subjects. SzAff subjects were also 6-7-times more likely to be given co-treatment with a mood-stabilizer or antidepressant. All subjects were rated at moderate symptomatic severity by PANSS and BPRS. Adherence to prescribed treatments was rated as good by DAI-30 score, and the risk of adverse effects was moderate at $19.8 \%$. 
TABLE 1 | Factors associated with metabolic syndrome in 151 psychotic disorder patients.

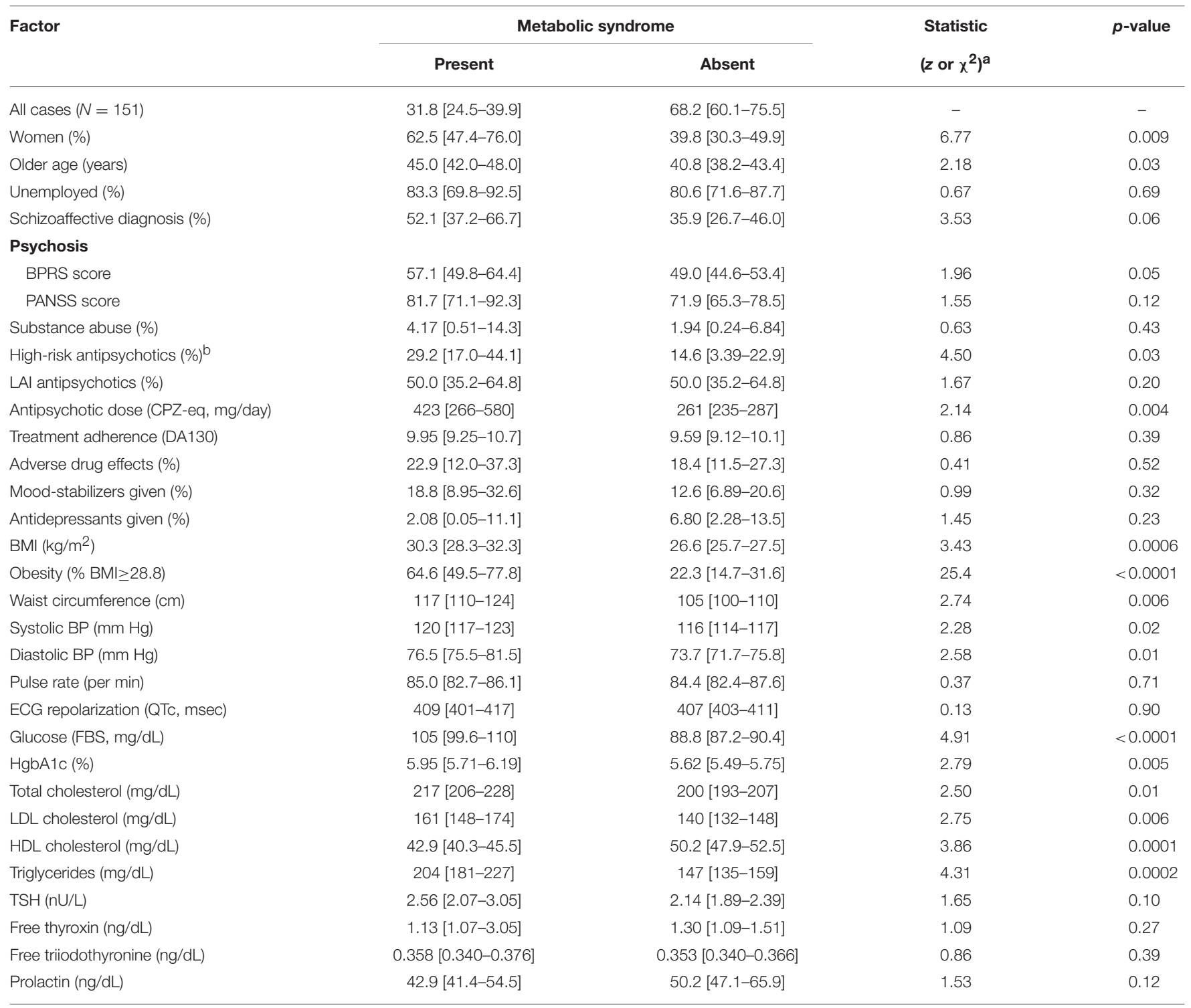

BPRS, Brief Psychiatric Rating Scale; PANSS, Positive and Negative Syndrome Scale; LAl, long-acting injectable antipsychotics; CPZ, chlorpromazine; DAl, Drug Attitude Inventory; $B M I$, body-mass index; BP, blood pressure; ECG, electrocardiogram; Hgb, hemoglobin; LDL, low-density lipoprotein; HDL, high-density lipoprotein; TSH, thyroid-stimulating hormone. ${ }^{a}$ Mann-Whitney (z-score) or contingency table $\left(x^{2}\right) .{ }^{b}$ Clozapine, olanzapine, paliperidone, quetiapine.

The overall prevalence of MetS (31.8\%) was moderate, but ranged from $18.2 \%$ with aripiprazole to $83.3 \%$ with quetiapine (Table 3). By comparison, reported prevalence of MetS in patients with psychotic disorders has ranged from 30 to $67 \%(1,7$, 20,21 ). Based on multivariate modeling, factors associated with MetS were antipsychotic dose, older age, and SzAff diagnosis, but not oral vs. LAI antipsychotics.

Of note, the present finding of significantly greater risk of MetS with SzAff vs, Sz diagnoses supports one hypothesis of this study, and adds to a previous suggestion of such a relationship (6). Although use of complex medication regimens was much more prevalent among SzAff subjects, these were infrequent and not significantly associated with risk of MetS. In addition, CPZ-eq antipsychotic doses were somewhat higher among SzAff subjects, although both factors appeared to operate somewhat independently (Table 4). Nevertheless, we suggest that the relative instability of SzAff disorders contributes to the use of more complex treatments (7) and this feature as well perhaps as intrinsic characteristics of such patients may contribute to risk of MetS.

In addition, contrary to prediction, we did not find a significant difference in risk of MetS in association with treatment with oral vs. LAI antipsychotics. This finding appears to be consistent with other recent reports indicating that LAI agents may not be safer than oral antipsychotics $(8,10)$. However, several measures tended to be less abnormal with LAI treatments (including lower total and LDL cholesterol, triglycerides and prolactin, smaller waist-circumference, and 
TABLE 2 | Multivariable logistic regression modeling: diagnostic measures associated with metabolic syndrome.

\begin{tabular}{lccc}
\hline Measure & OR [95\% Cl] & $\chi^{\mathbf{2}}$ & $\boldsymbol{p}$-value \\
\hline Higher FBS & $1.12[1.07-1.17]$ & 25.2 & $<0.0001$ \\
Lower HDL & $1.16[1.08-1.24]$ & 17.0 & $<0.0001$ \\
Diastolic blood pressure & $1.10[1.04-1.16]$ & 10.3 & 0.001 \\
Higher BMl & $1.17[1.06-1.29]$ & 9.53 & 0.002 \\
Female sex & $5.72[1.85-17.7]$ & 9.21 & 0.002 \\
\hline
\end{tabular}

Model fit $\chi^{2}=93,5, p<0.0001$. Not associated, age, LDL level, waist-circumference.

TABLE 3 | Risk of metabolic syndrome: antipsychotic agents and doses.

\begin{tabular}{lcc}
\hline Drug & $\begin{array}{c}\text { Metabolic Syndrome } \\
\text { Prevalence (\% [CI]) }\end{array}$ & $\begin{array}{c}\text { Daily dose } \\
\text { (CPZ-eq mg [CI]) }\end{array}$ \\
\hline Quetiapine & $83.3[35.9-99.6]$ & $229[89.9-368]$ \\
Clozapine & $60.0[14,7-94.7]$ & $615[196-1034]$ \\
Paliperidone & $34.0[21.5-48.3]$ & $298[265-330]$ \\
Olanzapine & $33.4[13.3-59.0]$ & $378[309-448]$ \\
Risperidone & $23.5[10.7-41.2]$ & $266[232-301]$ \\
Haloperidol & $18.2[2.28-51.8]$ & $259[197-321]$ \\
Aripiprazole & $18.2[5.19-40.3]$ & $121[95.4-146]$ \\
\hline
\end{tabular}

Risk of MetS is not significantly associated with CPZ-eq dose (Spearman $r_{s}=0.402$, $p=0.325)$, but the drugs differ overall $\left(\chi^{2}=13.4, p=0.04\right)$. Relatively high-risk drugs are quetiapine, clozapine, paliperidone and olanzapine.

TABLE 4 | Multivariable logistic regression modeling: risk factors associated with metabolic syndrome.

\begin{tabular}{lccc}
\hline Factor & OR [95\% Cl] & $\chi^{2}$ & $\boldsymbol{p}$-value \\
\hline Antipsychotic dose (CPZ-eq) & $1.003[1.001-1.005]$ & 4.80 & 0.028 \\
Older age & $1.03[1.01-1.07]$ & 4.76 & 0.029 \\
Diagnosis: Schizoaffective & $2.28[1.06-4.90]$ & 4.46 & 0.035 \\
Oral vs. LAl antipsychotics & $1.01[0.46-2.24]$ & 0.001 & 0.98 \\
\hline
\end{tabular}

Model fit $\chi^{2}=17.9, p=0.001$. Not associated, sex, diagnosis, psychosis severity. (BPRS rating).

shorter QTc interval). In addition, we found marked differences in risk of MetS between particular antipsychotic agents, with higher risk associated with quetiapine, clozapine, paliperidone,

\section{REFERENCES}

1. De Hert M, Dekker JM, Wood D, Kahl KG, Holt RI, Möller HJ. Cardiovascular disease and diabetes in people with severe mental illness position statement from the European Psychiatric Association (EPA), supported by the European Association for the Study of Diabetes (EASD) and the European Society of Cardiology (ESC). Eur Psychiatry (2009) 24:412-24. doi: 10.1016/j.eurpsy.2009. 01.005

2. Ventriglio A, Gentile A, Stella E, Bellomo A. Metabolic issues in patients affected by schizophrenia: clinical characteristics and medical management. Front Neurosci. (2015) 9:297-303. doi: 10.3389/fnins.2015.00297 and olanzapine, which are known to be associated with relatively high risks of weight-gain $(1,2,18,22,23)$.

\section{Limitations}

The study included a relatively small number of subjects and its findings may not generalize to other sites. Its cross-sectional design supports associations with MetS, but precludes causal inferences. In addition, estimates of CPZ-eq doses of LAI antipsychotics are not adjusted for probable but uncertain differences in bioavailability of injected vs. orally administered drugs.

\section{CONCLUSIONS}

This observational study of 151 patient-subjects with chronic psychotic disorders found a moderate prevalence of MetS (31.8\%), which was associated with being overweight or obese and with antipsychotic agents prone to leading to weight-gain, as well as higher antipsychotic CPZ-eq doses, and older age. Notably, risk of MetS was somewhat greater among SzAff than $\mathrm{Sz}$ subjects, but did not differ significantly between treatment with oral and LAI antipsychotics nor with the relatively infrequent use of adjunctive mood-stabilizers or antidepressants. However, several metabolic measures tended to be less abnormal among SzAff than Sz subjects. The association of MetS with SzAff (more than with Sz) probably reflects the complexity of SzAff disorders and their pharmacological treatment, including somewhat higher antipsychotic doses and more co-treatment with mood-stabilizers and antidepressants, but may also reflect other unknown characteristics of the disorders themselves.

\section{AUTHOR CONTRIBUTIONS}

$\mathrm{AV}, \mathrm{GV}, \mathrm{IB}, \mathrm{AC}, \mathrm{AR}$, and AP recruited patients and collected data. $\mathrm{AV}$ and $\mathrm{RB}$ wrote the paper. $\mathrm{AB}$ supervised clinical work and the manuscript drafting.

\section{FUNDING}

Supported by Department of Clinical and Experimental Medicine of University of Foggia. Supported, in part, by a grant from the Bruce J. Anderson Foundation and by the McLean Private Donors Psychopharmacology Research Fund (to RB).
3. De Hert M, Detraux J, Vancampfort D. The intriguing relationship between coronary heart disease and mental disorders. Dialog Clin Neurosci. (2018) 20:31-40.

4. Vancampfort D, Vansteelandt K, Correll CU, Mitchell AJ, De Herdt A, Sienaert P, et al. Metabolic syndrome and metabolic abnormalities in bipolar disorder: meta-analysis of prevalence rates and moderators. Am J Psychiatry (2013) 170:265-74. doi: 10.1176/appi.ajp.2012.120 50620

5. Ventriglio A, Gentile A, Baldessarini RJ, Martone S, Vitrani G, La Marca $\mathrm{A}$, et al. Improvements in metabolic abnormalities among overweight schizophrenia and bipolar disorder patients. Eur Psychiatry (2014) 29:402-7. doi: 10.1016/j.eurpsy.2013.11.005 
6. Bartoli F, Crocamo C, Caslini M, Clerici M, Carrà G. Schizoaffective disorder and metabolic syndrome: meta-analytic comparison with schizophrenia and other nonaffective psychoses. J Psychiatr Res. (2015) 66-67:127-34. doi: 10.1016/j.jpsychires.2015.04.028

7. Chouinard VA, Pingali SM, Chouinard G, Henderson DC, Mallya SG, Cypess $\mathrm{AM}$, et al. Factors associated with overweight and obesity in schizophrenia, schizoaffective and bipolar disorders. Psychiatry Res. (2016) 237:304-10. doi: 10.1016/j.psychres.2016.01.024

8. Gentile S. Safety concerns associated with second-generation antipsychotic long-acting injection treatment. A systematic update. Horm Mol Biol Clin Investig. (2017). doi: 10.1515/hmbci-2017-0004. [Epub ahead of print].

9. Brissos S, Ruiz Veguilla M, Taylor D, Balanzá-Martinez V. Role of long-acting injectable antipsychotics in schizophrenia: a critical appraisal Ther Adv Psychopharmacol. (2014) 4:198-219. doi: 10.1177/20451253145 40297

10. Sanchez-Martinez V, Romero-Rubio D, Abad-Perez MJ, Descalzo-Cabades MA, Alonso-Gutierrez S, Salazar-Fraile J, et al. Metabolic syndrome and cardiovascular risk in people treated with long-acting injectable antipsychotics. Endocr Metab Immune Disord Drug Targets (2018) 18:379-87. doi: 10.2174/1871530317666171120151201

11. American Psychiatric Association (APA). Diagnostic and Statistical Manual of Mental Disorders. 4th ed. Text Revision (DSM-IV-TR). Washington, DC: American Psychiatric Publishing (2000).

12. Kay SR, Fiszbein A, Opler LA. The positive and negative syndrome scale for schizophrenia (PANSS). Schizophrenia Bull. (1987) 13:261-76. doi: $10.1093 /$ schbul/13.2.261

13. Overall JE, Gorham DR. The Brief Psychiatric Rating Scale (BPRS). Psychol Rep. (1962) 10:799-812. doi: 10.2466/pr0.1962.10.3.799

14. Hogan TP, Awad AG, Eastwood R. Self-report scale predictive of drug compliance in schizophrenics: reliability and discriminative validity. Psychol Med. (1983) 13:177-83. doi: 10.1017/S00332917000 50182

15. Alberti KG, Zimmet P, Shaw J. Metabolic syndrome-a new worldwide definition: consensus statement from the International Diabetes Federation. Diabet Med. (2006) 23:469-80. doi: 10.1111/j.1464-5491.2006.0 1858.x
16. Alberti KG, Eckel RH, Grundy SM, Zimmet PZ, Cleeman JI, Donato KA, et al. Harmonizing the metabolic syndrome. Circulation (2009) 120:1640-5. doi: 10.1161/CIRCULATIONAHA.109.192644

17. Gardner D, Murphy A, Baldessarini RJ. Equivalent doses of antipsychotic agents: findings from an international Delphi survey. Am J Psychiatry (2010) 167:686-93. doi: 10.1176/appi.ajp.2009.09060802

18. Baldessarini RJ. Chemotherapy in Psychiatry. 3rd ed. New York, NY: Springer Press (2013).

19. Centorrino F, Sani G, Fogarty KV, Salvatore P, Talamo A, Cincotta SL, et al. Combinations of mood-stabilizers with antipsychotics as treatment strategies in hospitalized psychiatric patients. Clin Neuropsychiatry (2006) 3:322-26.

20. Centorrino F, Masters G, Baldessarini RJ, Öngür D. Metabolic syndrome in psychiatrically hospitalized patients treated with antipsychotics and other psychotropics. Hum Psychopharmacol. (2012) 27:521-6. doi: 10.1002/hup.2257

21. Correll CU, Frederickson AM, Kane JM, Manu P. Metabolic syndrome and the risk of coronary heart disease in 367 patients treated with second generation antipsychotic drugs. J Clin Psychiatry (2006) 67:575-83. doi: 10.4088/JCP.v67n0408

22. Musil R, Obermeier M, Russ P, Hamerle M. Weight-gain and antipsychotis; drug safety review. Expert Opin Drug Safety (2015) 14:73-96. doi: 10.1517/14740338.2015.974549

23. Cascade E, Kalali AH, Buckley P. Treatment of schizoaffective disorder. Psychiatry (2009) 6:15-7.

Conflict of Interest Statement: The authors declare that the research was conducted in the absence of any commercial or financial relationships that could be construed as a potential conflict of interest.

Copyright (C) 2019 Ventriglio, Baldessarini, Vitrani, Bonfitto, Cecere, Rinaldi, Petito and Bellomo. This is an open-access article distributed under the terms of the Creative Commons Attribution License (CC BY). The use, distribution or reproduction in other forums is permitted, provided the original author(s) and the copyright owner(s) are credited and that the original publication in this journal is cited, in accordance with accepted academic practice. No use, distribution or reproduction is permitted which does not comply with these terms. 